\title{
Metodologia de Baixo Custo para Levantamento de Diagramas CCT em Soldagem
}

\author{
(Low-Cost Methodology for Obtaining CCT Welding Diagrams)
}

\author{
Louriel Oliveira Vilarinho e Douglas Bezerra de Araújo ${ }^{1}$ \\ ${ }^{1}$ Laprosolda - Centro para Pesquisa e Desenvolvimento de Processos de Soldagem, Universidade Federal de Uberlândia, Uberlândia, \\ MG, Brasil,vilarinho@mecanica.ufu.br,douglas_ba@yahoo.com.br
}

\begin{abstract}
Resumo
A união de materiais metálicos é freqüentemente associada a problemas metalúrgicos, associados ao material em uso e que, cuja solução, demanda estudos dedicados e demorados. Especificamente, podem ocorrer modificações metalúrgicas na ZAC (zona afetada pelo calor), levando a alterações microestruturais. Contudo esta é uma região de difícil estudo devido a sua pequena dimensão. Assim, a fim de superar esta limitação com relação ao estudo da ZAC, existem na literatura proposições de máquinas de simulação de ZAC, que fazem uso do efeito Joule para o aquecimento de determinados corpos de prova, que se resfriam por condução e convecção, de forma a simular o ciclo térmico em uma ZAC obtida por soldagem real. Tais simuladores comerciais estão disponíveis, mas a um custo muito alto. Assim, o desenvolvimento de um simulador físico de baixo custo é de grande importância. Em artigos anteriores, a proposição e construção deste simulador, que está baseado na modificação de um equipamento de soldagem a ponto por resistência, foram avaliadas como uso de corpos de prova cilíndricos de geometria fixa. Embora, a fonte consiga suprir a energia necessária para se atingir o ciclo térmico de aquecimento (alta temperatura e baixo tempo), não foi possivel atingir um ciclo térmico de resfriamento próximo ao da realidade. Assim, para suprir tal limitação, simulações foram executadas em elementos para determinar a geometria ótima em função do tempo de resfriamento (ciclo térmico) desejado. Todos os dados das simulações foram compilados na forma de uma modelo de regressão para se executar tal otimização da geometria. A partir desta metodologia foi possível construir diferentes diagramas CCT aplicados obtidos com os rápidos ciclos térmicos presentes na soldagem. Tais diagramas são apresentados e comparados com os poucos encontrados na literatura, com boa concordância.
\end{abstract}

Palavras-chave: Modelamento; Método dos Elementos Finitos; Simulação Física.

Abstract: The joining of metals is often associated to metallurgical problems, which is specific for each material and, therefore, demands dedicated and time-consuming study. Specifically, metallurgical transformations in the HAZ (Heat Affected Zone) can occur and lead to microestructural changes such as grain growth, fragile structures presence, cracking, among others. The HAZ is a difficult region for studying due to its small dimensions, where its properties are defined basically by the base metal characteristics and the factors that affect the thermal cycle of the process. Thus, in order to overcome this limitation of the HAZ study, there are in the literature different proposes of HAZ-simulator machines, which uses the Joule effect for heating specific coupons that cool down by conduction and convection. This approach intends to simulate the thermal cycle in a "real" HAZ obtained in a "real" welding. Comercial simulators are available at very high costs. Therefore, the development of a low-cost machine is welcome. In a previous study, the proposition and construction of this machine, based on the modification of resistance spot welding equipment, was carried out and the first coupons were fabricated in a cylindrical geometry. Although the machine provides sufficient energy, the use of traditional coupons with cylindrical geometry presents restrictions of portraying the "real" case, i.e., the welding. In the cylindrical geometry option, the obtained thermal cycles do not present temperature gradients closer to the ones in "real" weldments. Hence, to overcome this limitation, finite elements modelling was carried out and different coupon geometries were simulated. The objective is to reach thermal cycles as close as possible to the ones obtained in a "real" situation. A database of different geometrical features was achieved by using the finite element analyses. This database was used as the input of model regression analyses by using statistical ANOVA. The empirical resulting model was employed as the input of an optimization algorithm, which is used for searching a given cooling rate desired by the user. From these results is possible to obtain different cooling rates and build-up Continuous Cooling Transformation (CCT) Diagrams dedicated to welding, i.e., with higher cooling rates. Comparison was found in good agreement between obtained diagrams and the literature ones.

Key-words: Modeling; Finite Element Technique; Physical Simulation.

(Recebido em 29/03/2010; Texto final em 23/07/2010).

Artigo originalmente publicado no CONSOLDA 2009,

Piracicaba, SP, Outubro de 2009. 


\section{Introdução}

Durante o processo de soldagem pode ocorrer o aparecimento de alguns problemas como o crescimento de grãos, aparecimento de estruturas frágeis e fissurações, dentre outros [1-5]. Tais problemas metalúrgicos são freqüentemente associados à zona afetada pelo calor (ZAC) [6-9] de dimensões reduzidas. Assim, o estudo das transformações microestruturais e conseqüentemente o estudo do ciclo térmico destas regiões torna-se ainda muito relevante.

Tais transformações microestruturais afetam diretamente as propriedades mecânicas, as tensões internas e deformações geométricas do material. Assim, uma ferramenta importante no estudo destas transformações microestruturais seria 0 diagrama de resfriamento continuo (CCT), que indica o tipo de microestrutura e sua quantidade para cada taxa de resfriamento. Entretanto, a disponibilidade de diagramas CCT na literatura técnica é restrita, principalmente os diagramas CCT para soldagem. Tais diagramas voltados para soldagem diferem dos diagramas de tratamentos térmicos, pois o material é austenitizado a temperaturas mais altas e com taxas de aquecimento muito alto.

Desta forma, torna-se relevante um meio de se obter os diagramas CCT de soldagem, principalmente no modelamento numérico de tensões, deformações e transformações microestruturais dos processos de soldagem. Mais do que isto, em face à necessidade constante de redução de custos no meio industrial e o momento econômico atual, este trabalho tem como objetivo mostrar um método de baixo custo para a obtenção destes diagramas CCT através do estudo dos ciclos térmicos de corpos de prova de diferentes geometrias, onde estes corpos de provas são aquecidos por efeito Joule e resfriados por meio de condução e convecção natural.

\section{Procedimento Experimental}

O procedimento experimental (Figura 1) consta no aquecimento do corpo de prova por meio de uma fonte de soldagem a ponto adaptada, que fornece energia elétrica, que por efeito Joule aquecerá o corpo de prova até a temperatura normalmente alcançada na região da ZAC. Foram realizadas as aquisições de temperatura por meio de termopar do tipo $\mathrm{K}$ posicionado no centro simétrico do corpo de prova. O sinal do termopar passa por um amplificador de sinais e depois para um sistema de aquisição e para o controlador, que tem a função de desligar a fonte quando se atingir a temperatura de pico desejada.

\section{Testes Experimentais}

Foram utilizados nos experimentos diferentes geometrias de corpos de provas (Figura 2), com a finalidade de se conseguir diferentes taxas de aquecimentos e resfriamento. Estas geometrias foram analisadas primeiramente numericamente através do método de elementos finitos [10].

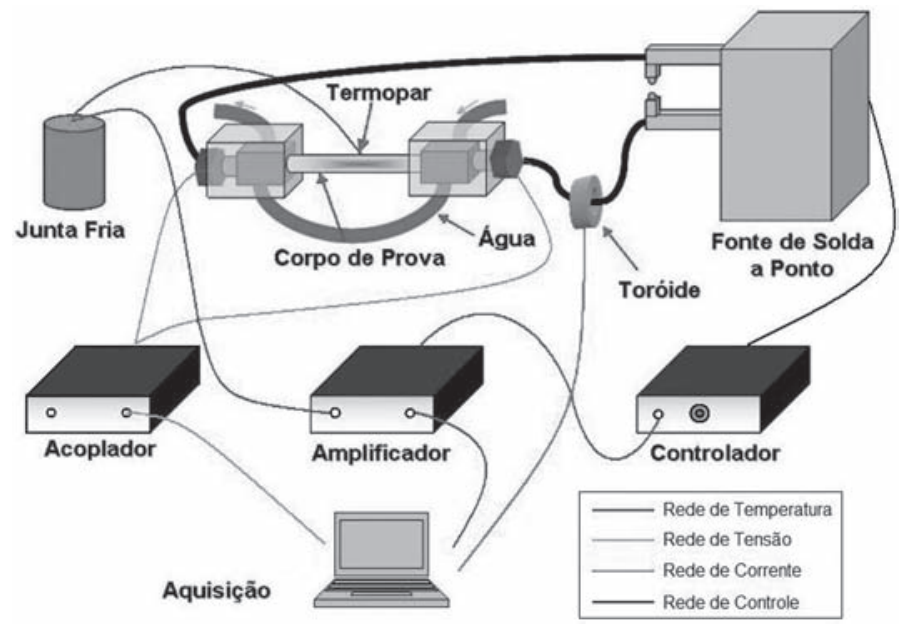

Figura 1. Montagem do equipamento simulador de ZAC

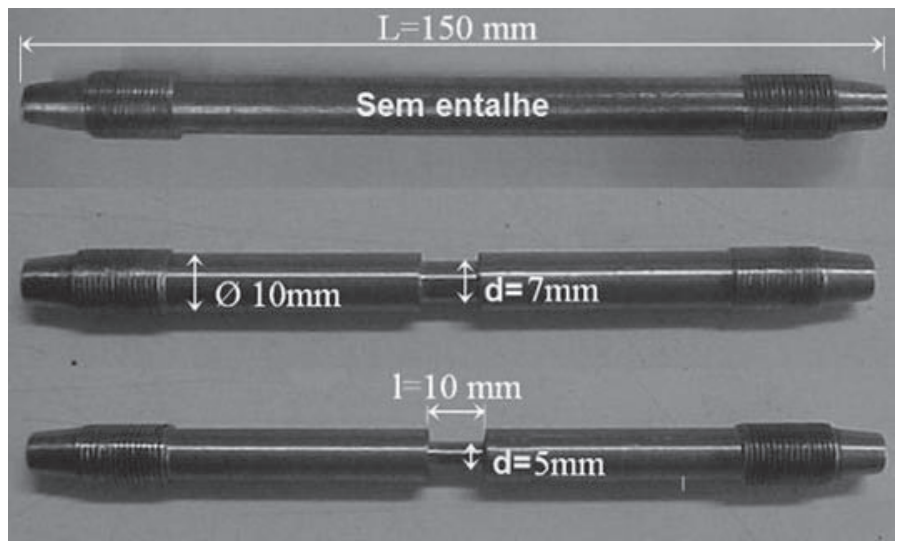

Figura 2. Diferentes geometrias de corpos de prova utilizados no experimento

Depois de realizados os experimentos com o simulador de ZAC e realizada a aquisição da temperatura, pode-se verificar a diferença no ciclo térmico entre os corpos de prova de diferentes geometrias. Como exemplo, a Figura 3 mostra visualmente a diferença no aquecimento entre dois corpos de prova de geometria diferente. A partir da aquisição do ciclo térmico, podese, por exemplo, verificar a diferença no tempo de aquecimento até $1350^{\circ} \mathrm{C}$ e o tempo de resfriamento entre $800^{\circ} \mathrm{C}$ e $500^{\circ} \mathrm{C}$, como características importantes em uma soldagem

\section{Levantamento dos Diagramas CCT}

Como metodologias para a determinação de transformações microestruturais cita-se a dilatometria, muito empregada [1113], análise térmica [14] e análise por resistência elétrica [15].

Normalmente, a natureza das transformações de fases é exotérmica ou endotérmica, sendo que estas variações térmicas podem ser registradas através de um termopar. Desta forma, traçando-se o gráfico de temperatura em função do tempo podem-se perceber as transformações de fase por um pequeno desvio da curva, conforme mostra a Figura 4.

Muitas vezes, as transformações são muito discretas e difíceis de perceber através de apenas observação. Com a finalidade de 

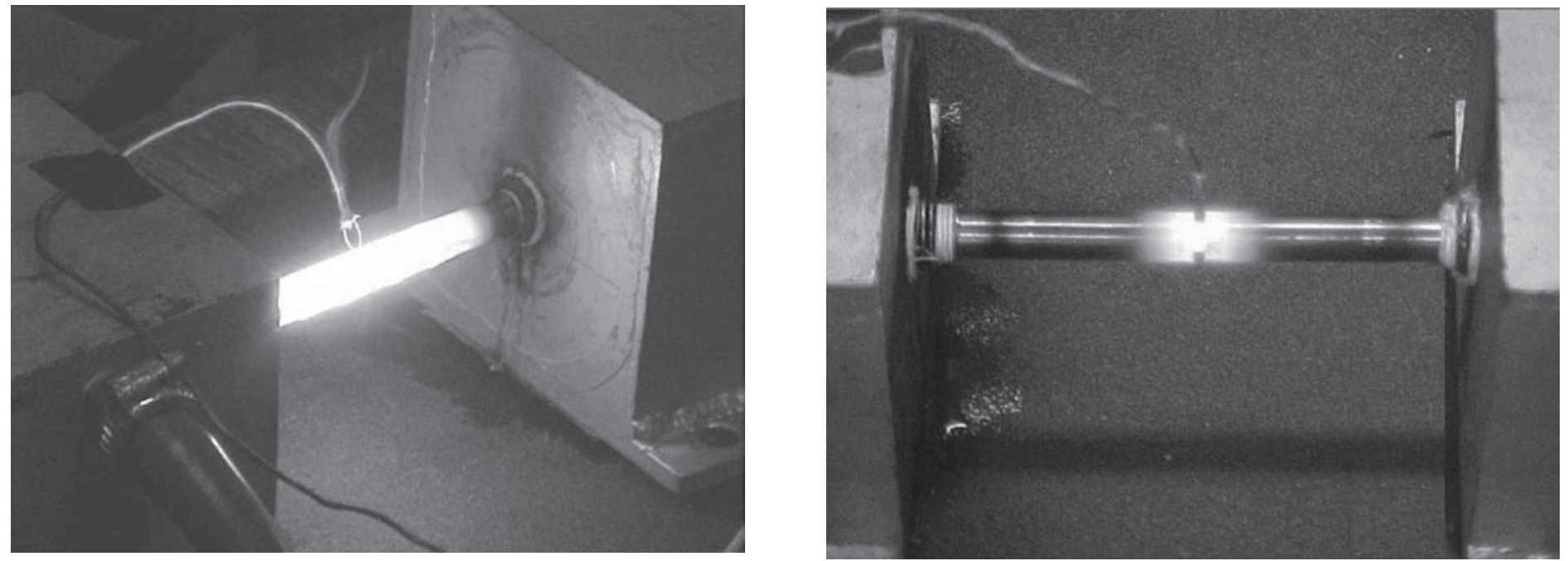

Figura 3. Corpos de prova sem e com rebaixo no momento do aquecimento

encontrar tais desvios na curva de aquecimento e resfriamento são utilizados alguns artifícios, sendo um deles a análise diferencial. Esta análise seria um método em que se utiliza uma curva de referência que é gerada através de uma função de regressão de partes da curva onde se queira analisar as transformações. Fazendo a diferença entre a curva de temperatura e a curva de referência (Equação 1), pode-se verificar com maior nitidez as transformações de fases [14].

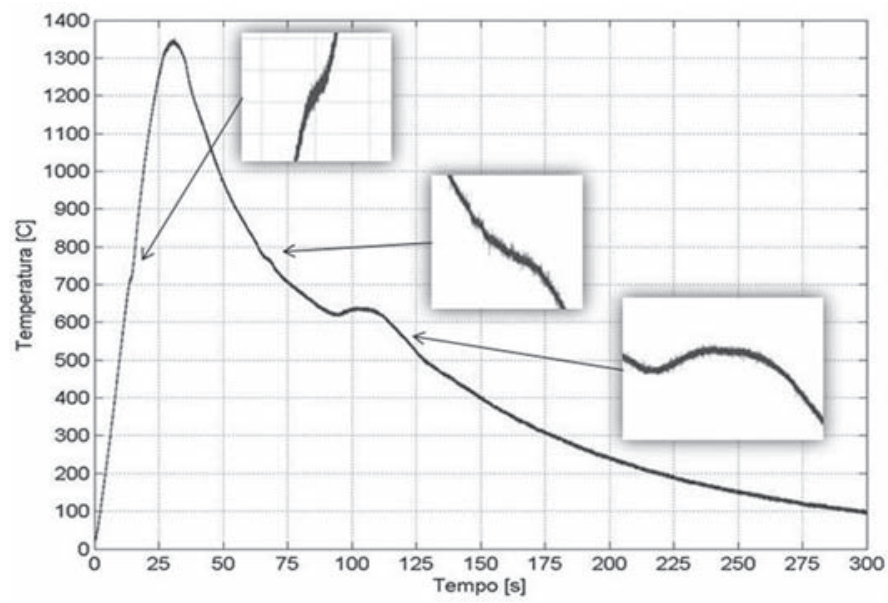

Figura 4. Detecção de algumas transformações de fase no aço AISI 1045

$$
\mathrm{D}(\mathrm{T})=\mathrm{T}-\mathrm{T}_{\mathrm{ref}}
$$

onde $T$ é a temperatura medida experimentalmente e $T_{r e f}$ é a temperatura obtida pela curva de referência dada pela Equação 2.

Já com relação à curva de referência, seria uma curva de regressão em que tem por objetivo tornar mais fácil a detecção das transformações de fase. Essa regressão é realizada em parte da curva de resfriamento experimental e é extrapolada para uma boa parte da curva conforme mostra a Figura 5.
A regressão foi do tipo exponencial (Equação 2), pois foi a que obteve o menor erro quadrático. Depois de realizada a regressão, fez-se a diferença entre a curva do ciclo térmico experimental e a curva de regressão (Figura 6), obtendo assim, possíveis regiões da curva em que há descontinuidades, sendo endotérmica ou exotérmica a determinada temperatura, mostrando desta forma as transformações microestruturais.

$$
\mathrm{T}_{\text {ref }}=\mathrm{a} \mathrm{e}^{\mathrm{bt}}+c \mathrm{e}^{\mathrm{dt}}
$$

onde $a, b, c, d$ são parâmetros de ajuste obtidos pela regressão.

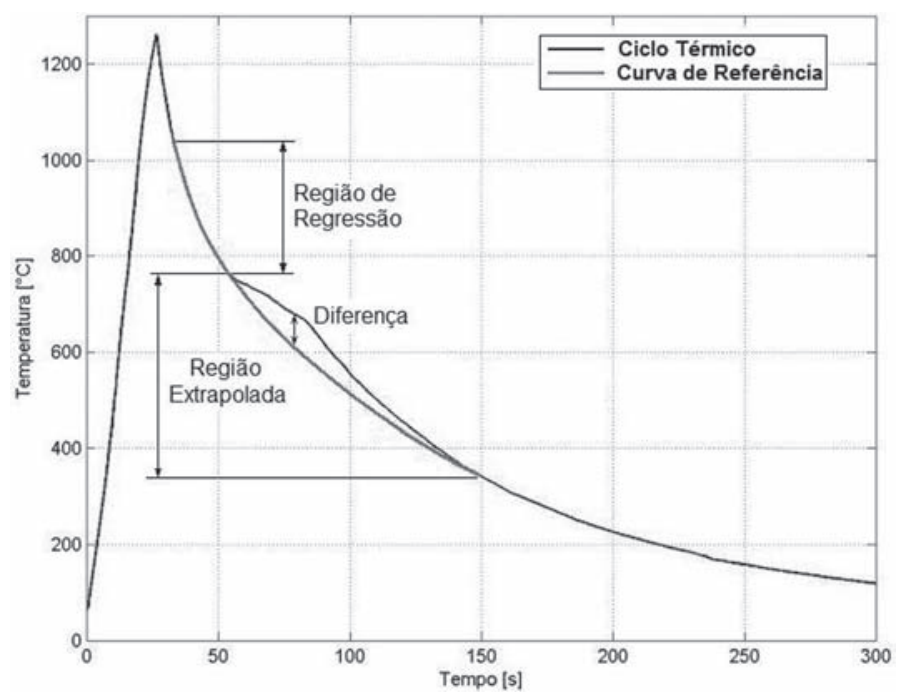

Figura 5. Demonstração da obtenção da curva de referencia

Assim, pela Figura 6, é possível se identificar a que temperatura e em que instante estão ocorrendo as transformações microestruturais no material.

\section{Resultados e Discussões}

Foram realizados experimentos com o aço AISI 1045 com a composição nominal mostrada na Tabela 1. 
Tabela 1 - Faixa de composição nominal do aço AISI 1045

\begin{tabular}{c|c|c|c|c}
\hline $\mathrm{Fe}$ & $\mathrm{C}$ & $\mathrm{Mn}$ & $\mathrm{P}$ & $\mathrm{S}$ \\
\hline $98,51-98,98 \%$ & $0,42-0,5 \%$ & $0,6-0,9 \%$ & $<0,04 \%$ & $<0,05 \%$ \\
\hline
\end{tabular}

Tabela 2. Valores das taxas de resfriamento entre as temperaturas de $800^{\circ} \mathrm{C}$ e $500^{\circ} \mathrm{C}$

\begin{tabular}{c|c|c|c|c}
\hline $\mathrm{d}[\mathrm{mm}]$ & $\mathrm{t} 800$ & $\mathrm{t} 500$ & $\mathrm{Dt} 8 / 5[\mathrm{~s}]$ & $\mathrm{f}_{8 / 5}\left[{ }^{\circ} \mathrm{C} / \mathrm{s}\right]$ \\
\hline 5 & 5.09 & 7.21 & 2.12 & 141.5 \\
\hline 6 & 10.93 & 20.74 & 9.81 & 30.6 \\
\hline 7 & 20.50 & 53.82 & 33.32 & 9.00 \\
\hline 8 & 53.08 & 125.60 & 72.52 & 4.10 \\
\hline 9 & 71.48 & 155.30 & 83.82 & 3.60 \\
\hline 10 & 100.20 & 190.60 & 90.40 & 3.30 \\
\hline
\end{tabular}

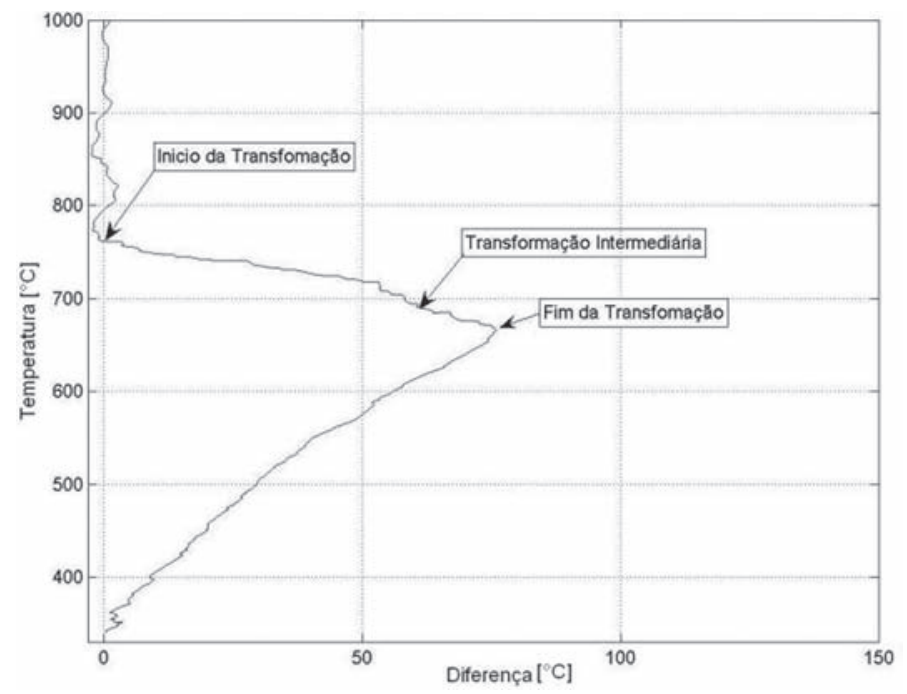

Figura 6. Curva da diferença entre a curva de resfriamento e a curva de referencia (regressão)

Aquecendo os corpos de prova do material com o diâmetro central de $5 \mathrm{~mm}$ a $10 \mathrm{~mm}$ e comprimento central fixo em 10 $\mathrm{mm}$ até uma temperatura de $1350^{\circ} \mathrm{C}$ e deixando resfriar-se naturalmente, foram obtidos diferente taxas de resfriamentos variando entre $3,3{ }^{\circ} \mathrm{C} / \mathrm{s}$ até $141,5^{\circ} \mathrm{C} / \mathrm{s}$. Este valores podem ser visto na Tabela 2. Já as curvas de resfriamentos para cada geometria podem ser visto por meio da Figura 7. Depois dos experimentos realizados, os corpos de prova foram seccionados ao centro, lixado, polido e atacados com Nital $5 \%$. As fotos das microestruturas de cada corpo de prova podem ser vistos na Figura 8.

Pela Figura 8, pode-se verificar através da microestrutura de três diferentes taxas de resfriamento. Para a taxa de resfriamento de $3,3^{\circ} \mathrm{C} / \mathrm{s}$ os grãos são de tamanhos maiores do que os grãos do corpo de prova com a taxa de resfriamento de $9^{\circ} \mathrm{C} / \mathrm{s}$. Isso é devido ao tempo de permanência acima da temperatura de $1100^{\circ} \mathrm{C}$ [6]. Já para o corpo de prova com a taxa de resfriamento de $141,5^{\circ} \mathrm{C} / \mathrm{s}$ a microestrutura resultante foi de martensita.

Utilizando o método da análise diferencial, descrito no item anterior, pode-se verificar as temperaturas nas quais

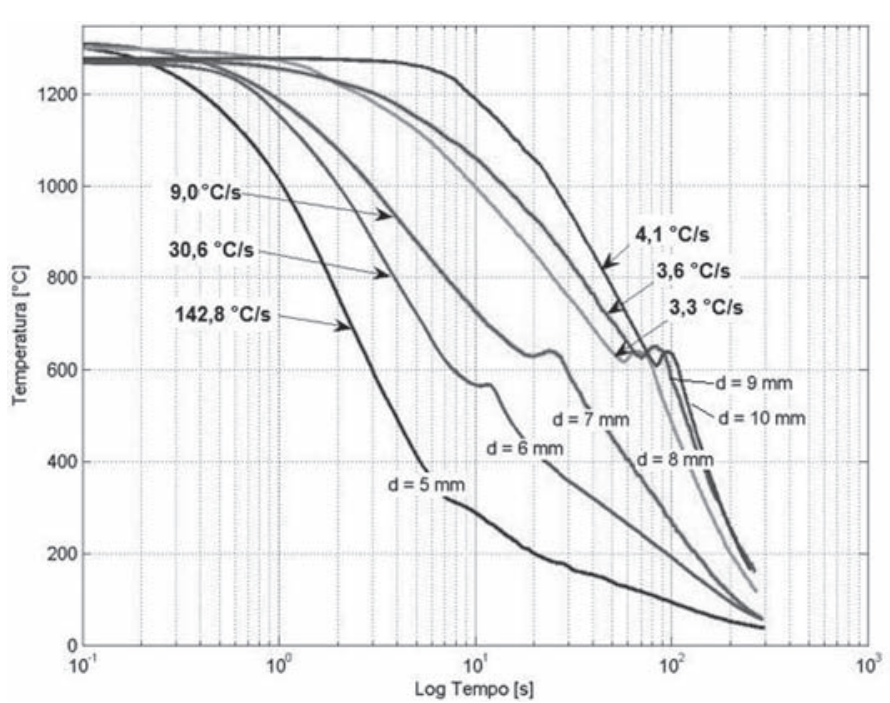

Figura 7. Curva de resfriamento para as diferentes geometrias de corpos de prova

estão ocorrendo as transformações microestruturais para cada geometria de corpo de prova. Através da Figura 9, pode-se ver o resultado utilizando as Equações 1 e 2 para algumas das geometrias de corpos de prova utilizadas.

Já de posse das temperaturas em que começam e das temperaturas em que terminam as transformações de fase e das micrografias dos diferentes corpos de prova é possível fazer a confecção do diagrama CCT de soldagem para este material, como mostra a Figura 10. Nesta figura é feita a comparação entre o diagrama obtido (em colorido) e o diagrama do aço AISI 1045 para tratamento térmico. A necessidade de tal comparação reflete a disponibilidade restrita dos diagramas CCT para soldagem, ou seja, mesmo se utilizando um aço comum como o AISI 1045 não foi possível encontrar diagramas CCT específico para soldagem e, portanto, a comparação foi feita com um diagrama voltado para tratamento térmico.

A diferença entre os diagramas ressalta o fato da maior temperatura de austenitização e maiores velocidades de resfriamento obtidas no caso da soldagem, o que desloca o diagrama obtido para a soldagem para a direita, mostrando a sensibilidade e eficácia da metodologia de baixo custo proposta. 
Diâmetro Central $=10 \mathrm{~mm}$ e $\phi_{8 / 5}=3.3^{\circ} \mathrm{C} / \mathrm{s}$

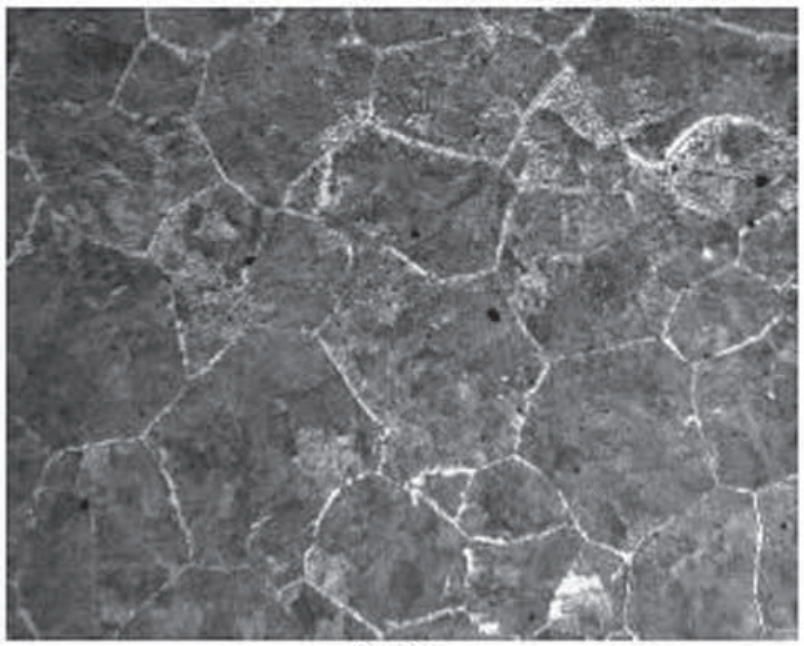

$100 x$

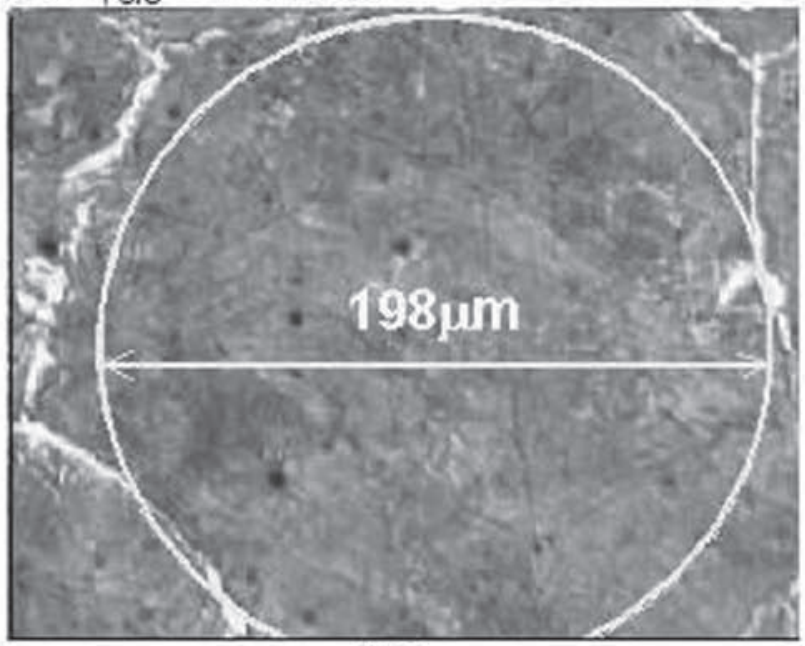

$400 x$

Diâmetro Central $=7 \mathrm{~mm}$ e $\phi_{8 / 5}=9^{\circ} \mathrm{C} / \mathrm{s}$

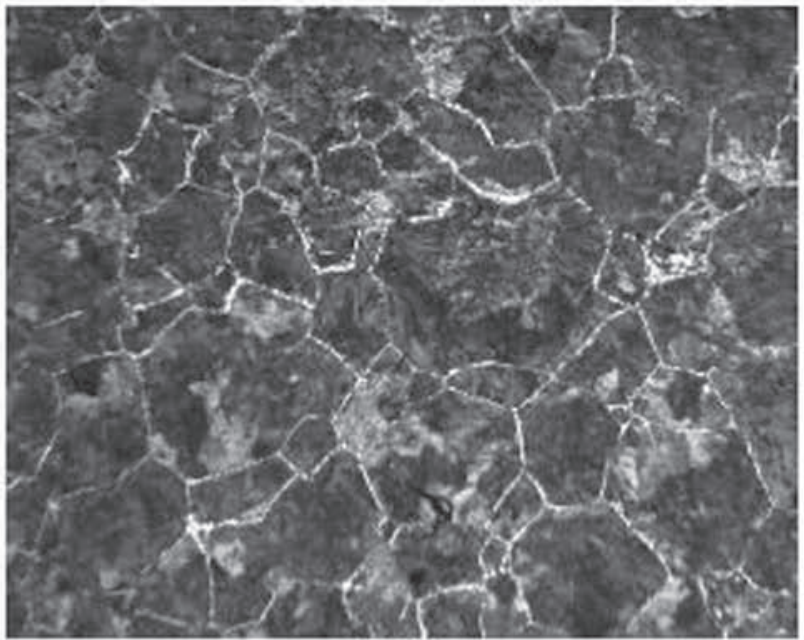

$100 x$

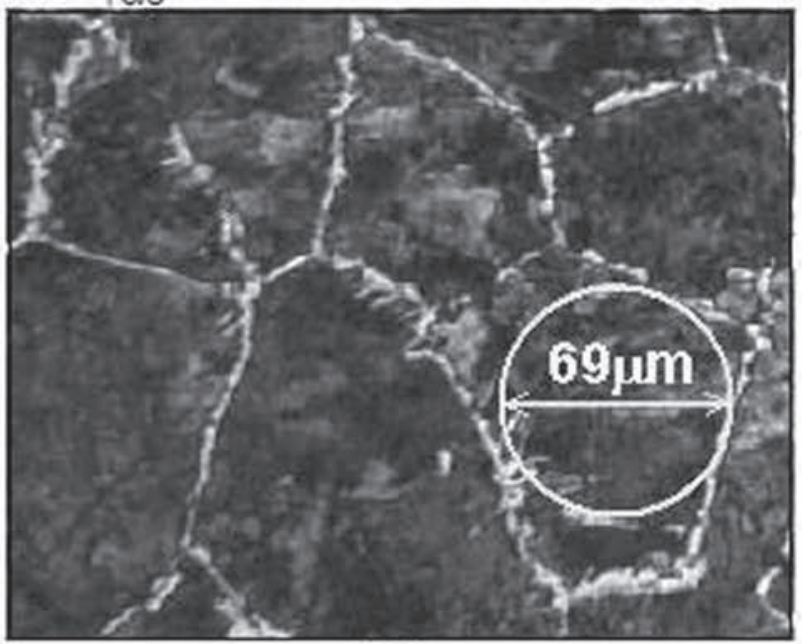

$400 x$

Diâmetro Central $=5 \mathrm{~mm}$ e $\phi_{8 / 5}=142,8^{\circ} \mathrm{C} / \mathrm{s}$

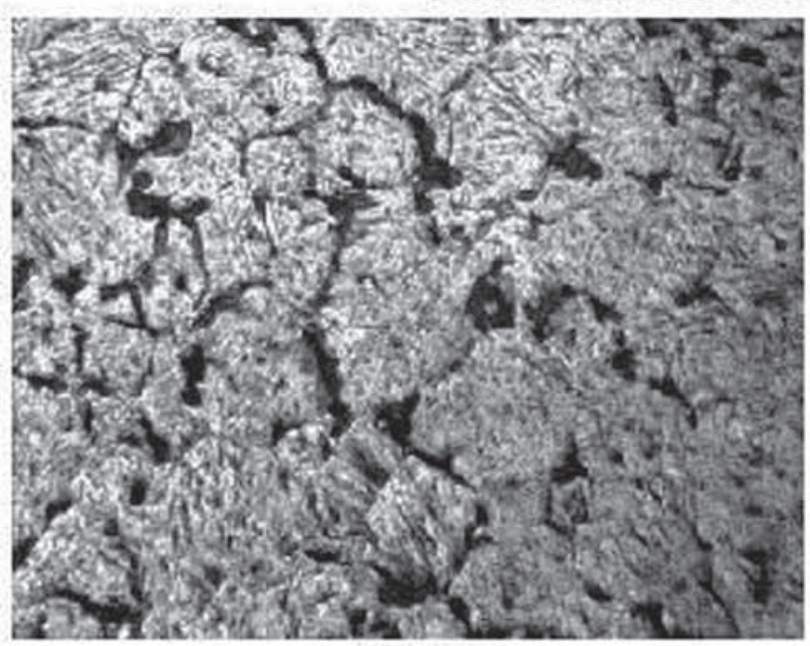

$100 x$

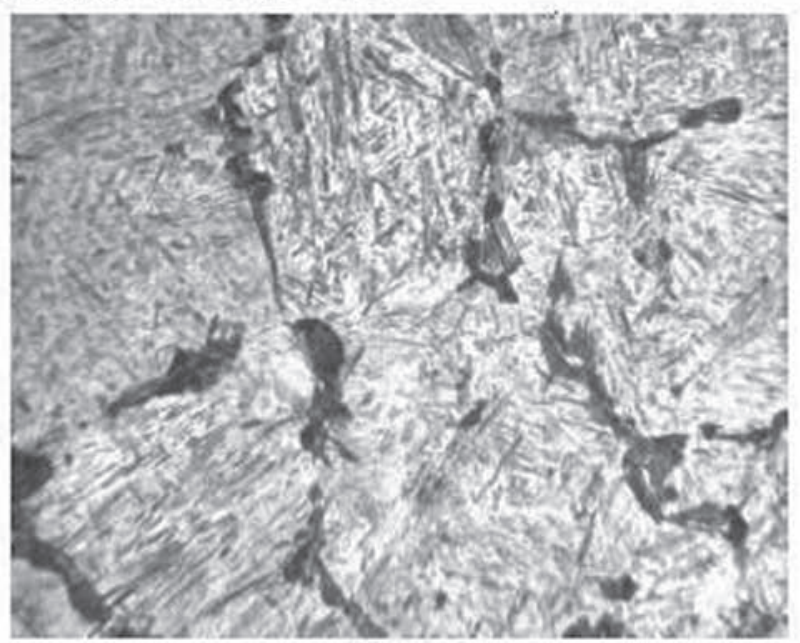

$400 x$

Figura 8. Microestrutura do aço AISI 1045 para as diferentes taxas de resfriamento 


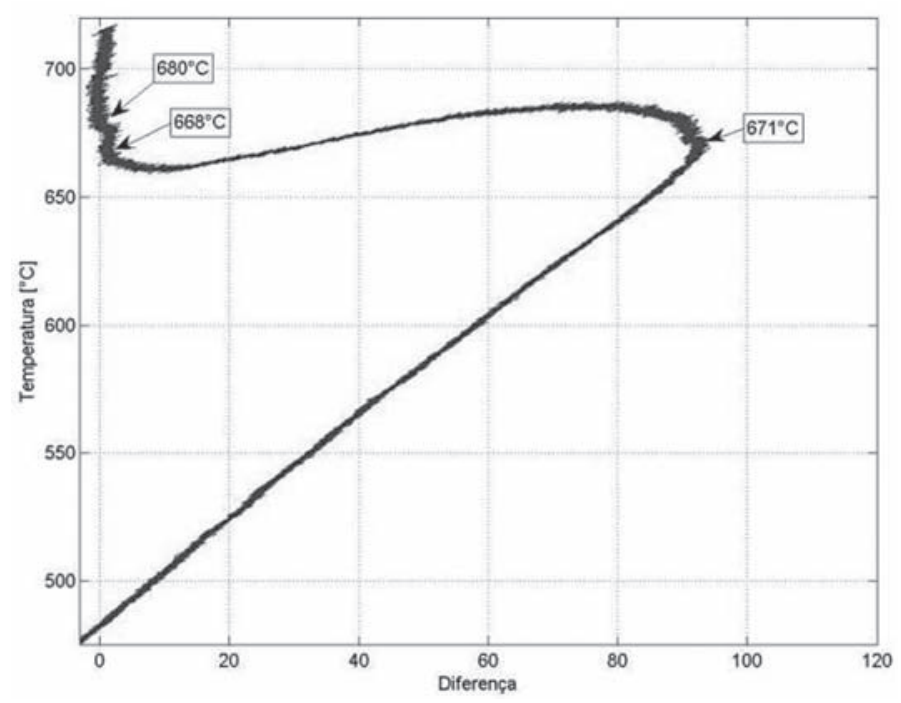

$\mathrm{d}=10 \mathrm{~mm}-\phi_{8 / 5}=3.31^{\circ} \mathrm{C} / \mathrm{s}$

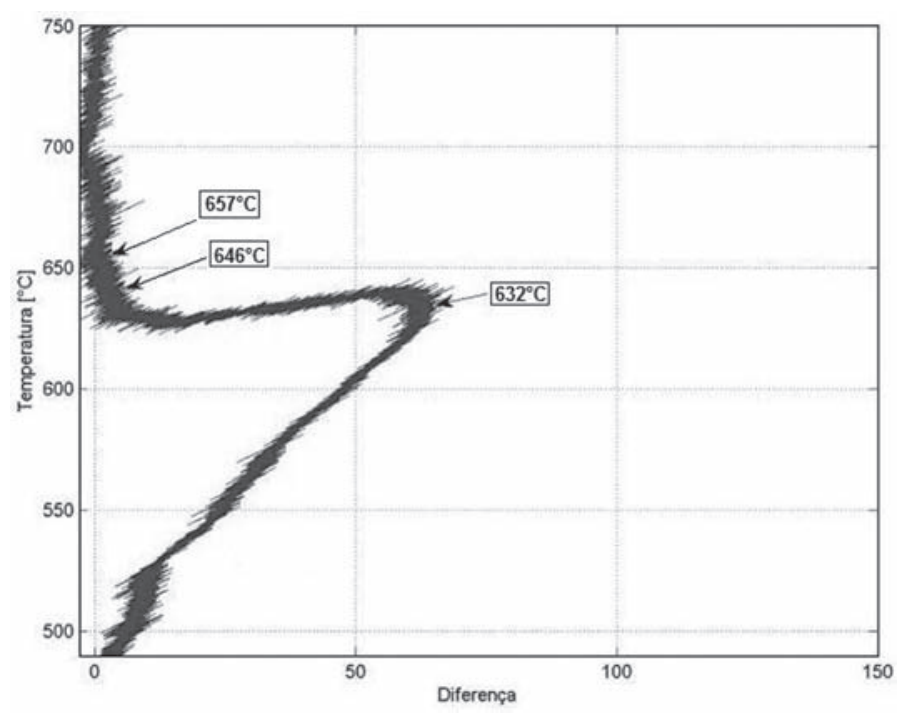

$\mathrm{d}=7 \mathrm{~mm}-\phi_{8 / 5}=8.94^{\circ} \mathrm{C} / \mathrm{s}$

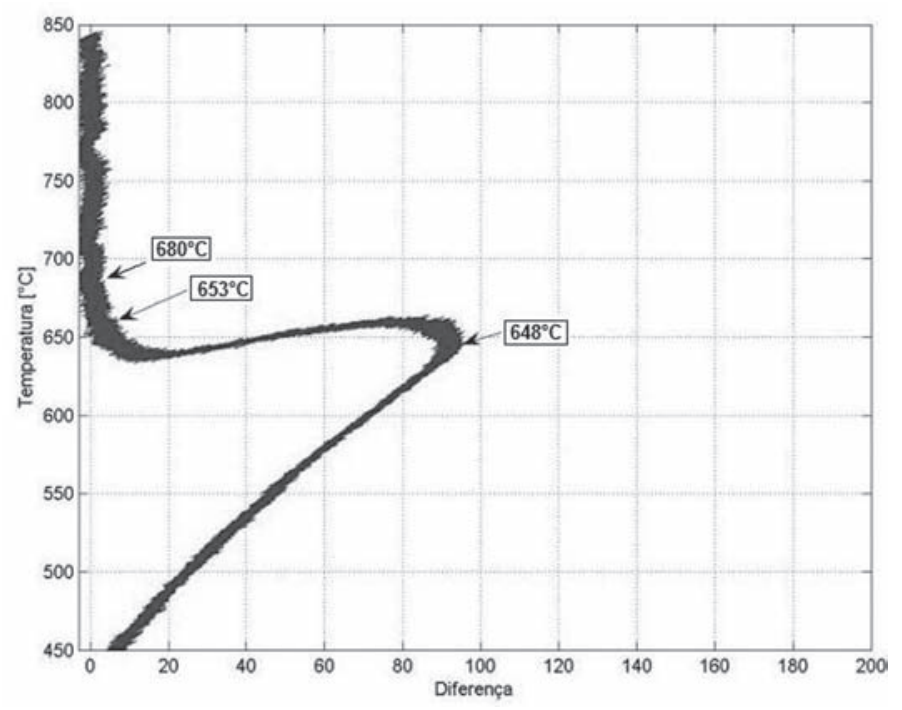

$\mathrm{d}=8 \mathrm{~mm}-\phi_{8 / 5}=4.13^{\circ} \mathrm{C} / \mathrm{s}$

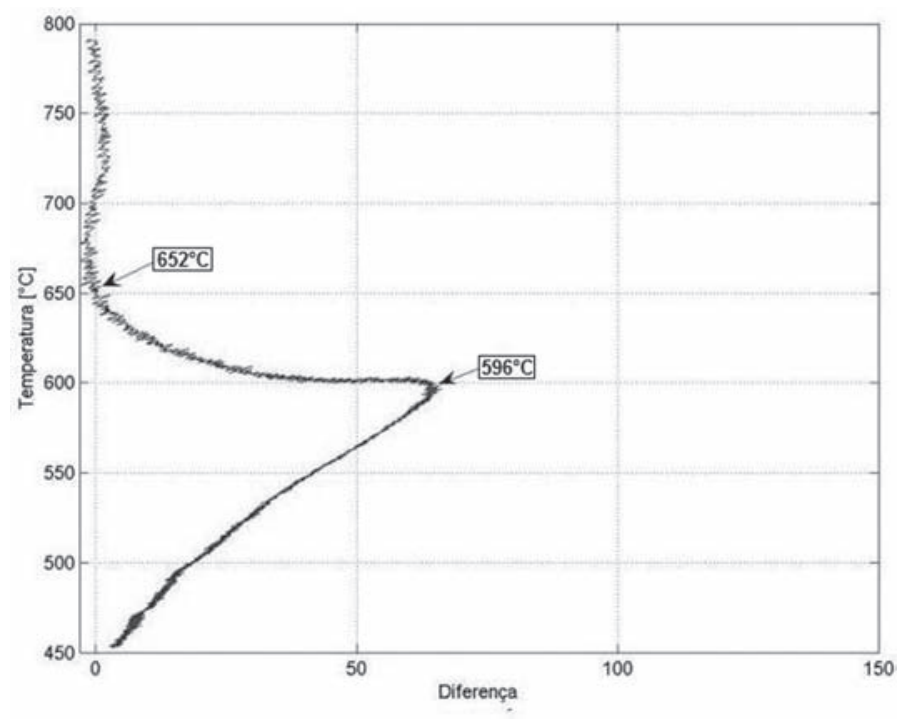

$\mathrm{d}=6 \mathrm{~mm}-\phi_{8 / 5}=27.9^{\circ} \mathrm{C} / \mathrm{s}$

Figura 9. Detecção das transformações microestruturais e das temperaturas em que elas se iniciam e terminam

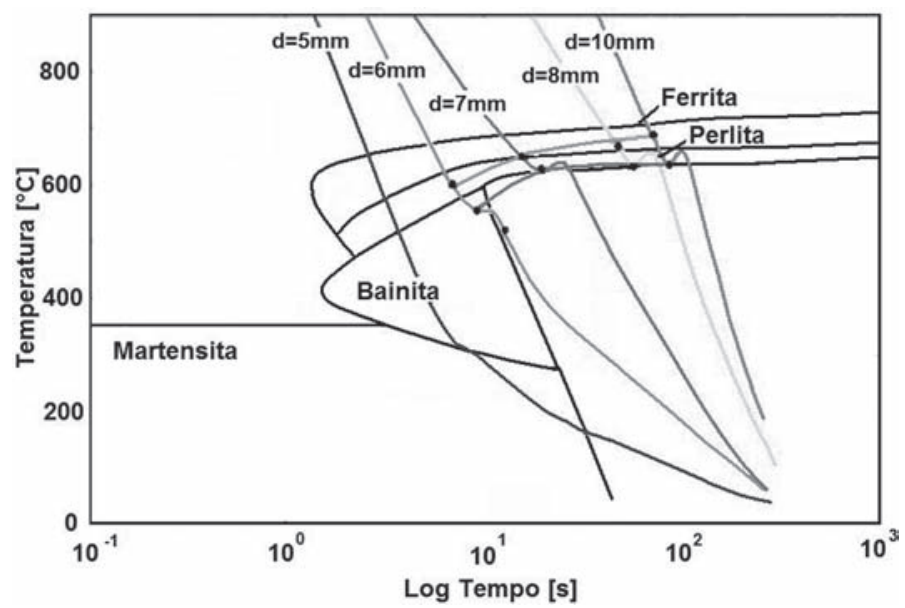

Figura 10. Comparação entre os diagramas CCT de tratamento térmico (preto) e o diagrama CCT de soldagem (colorido)

\section{Conclusão}

A partir do simulador de ZAC e da análise diferencial do histórico térmico de corpos de prova, foi possível obter diagrama CCT específico para a soldagem via metodologia de baixo custo.

\section{Agradecimentos}

Os autores gostariam de agradecer à Fapemig, Capes e CNPq pelo auxílio no financiamento de bolsas e consumíveis utilizados no trabalho.

\section{Referências Bibliográficas}

[1] TOMITA, Y. et al. Improvement in HAZ Toughness of Steel by TinN-MnS Addition. ISIJ International, 34, 829-235, p. 1994. 
[2] FANG, D., LU, Z., \& HE, J. Examination of a Crack Found in a Welded Joint of Steel TTStE36 after 13 Years Service . Internation Journal of Pressure Vessels and Piping, p. 571-573, 1999.

[3] OMWEG, G. M. et al. Effect of Welding Parameters and H2S Partioal Pressure on the Susceptibility of Welded HSLA Steel to Sulfide Stress Cracking. Welding Journal, p. 136144, 2003.

[4] RAMIREZ, J. E., MISHAEL, S., \& SHOCKLEY, R. Properties and Sulfide Stress Cracking Resistance of CoarseGrained Heat-Affected Zones in V-Microalloyed X60 Steel Pipe. Welding Journal, p. 113-123, 2005

[5] CHOPRA, O. K. Crack Growth Rates of Irradiated Austenitic Stainless Steel Weld Heat Affected Zone in BWR Environments. U.S. Nuclear Regulatory Commission, Division of Engineering Technology, Washington, 2006.

[6] IKAWA, H., OSHIGE, H., \& NOI, S. Austenite Grain Growth of Steel in Weld-Heat Affected Zone. Transactions of the Japan Welding Society, 8, p. 52-57, 1977.

[7] MABUCHI, H., UEMORI, R., \& FUJIOKA, M. The Role of Mn Depletion in Intra Granular Ferrite Transformation in the Heat Affected Zone of Welded Joints with Large Heat Input in Structural Steels. ISIJ International, 36, p. 1406-1412, 1996.

[8] CARROUGE, D., \& BHADESHIA, H. K. Microstructural Change in High Temperature Heat- Affected Zone of Low Carbon Weldable "13\%Cr" Martensitic Stainless Steels. Stainless Steel World America, p. 16-23, 2002.

[9] MEADOWS, C., \& FRITZ, J. D. Understanding Stainless Steel Heat-Affected Zones. Welding Journal, p. 26-30, 2005.

[10] ARAÚJO, D. B. Otimização de Corpos de Prova para Utilização em Simulador de ZAC por Meio de Elementos Finitos. Dissertação de Mestrado. Universidade Federal de Uberlândia, 2008.

[11] ZHANG, Z., \& FARRAR, R. A. An Atlas of Continuous Cooling Transformation (CCT) Diagrams Applicable to Low Carbon Low Alloy Weld Metals, 1995.

[12] ANDRÉS, C. G. et al. Application of dilatometric analysis to the study of solid-solid phase transformations in steels. Materials Characterization, 48, p. 101-111, 2002.

[13] ALVES, C. H. Análise da Influencia da Taxa de Resfriamento no Gradiente Microestrutural de Barras Laminadas a Quente Tratadas Termicamente. Curitiba, 2004.

[14] ZACHRISSON, J. In Situ Detection and Characterisation of Phase Transformations in Weld Metals. Master's Thesis, 2006.

[15] PISTORIUS, P. G., \& ROOYEN, G. T. Detecting Phase Transformation in Electrically Conductive Materials by Means of Thermoeletric Anomalies, 1991.

[16] Matweb AISI 1045 Steel. Disponível em: <http://www. matweb.com>. Acesso em: junho 2008.

[17] IKAWA, H., et al. Austenite Grain Growth of Steel during Thermal Cycles. Transactions of the Japan Welding Society, 8, p. 46-51, 1977. 